\title{
To scan or not to scan: neuroimaging in mild cognitive impairment and dementia
}

\author{
Victoria Sullivan, Biswadeep Majumdar, Anna Richman \& Sobhan Vinjamuri
}

\begin{abstract}
SUMMARY
There is currently a huge variation in clinical practice as to whether patients being assessed for dementia undergo neuroimaging investigations. With an ageing population it is likely that there will be greater pressures on psychogeriatric services, so accurate assessment, diagnosis and prompt treatment will be required. This article will examine the evidence for the use of different neuroimaging techniques in the diagnosis of mild cognitive impairment and dementia.
\end{abstract}

\section{DECLARATION OF INTEREST}

None.

The UK has an ageing population; between 1992 and 2010 the percentage of the population aged over 65 years rose from 15.8 to $17.2 \%$ (an addition of about 870000 people) and by 2034 it is estimated that it will increase to $23 \%$. The most substantial rise has been in people over the age of 85 , increasing from 660000 in 1984 to 1.5 million in 2010 (Office for National Statistics 2012). Dementia is one of the most common conditions in the elderly and it is estimated that approximately 750000 people in the UK are affected. This number is expected to double over the next 30 years, which will increase financial pressures on clinical budgets, so there is a significant emphasis on early assessment, diagnosis and prompt initiation of treatment.

Computed tomography (CT) and magnetic resonance imaging (MRI) are widely used in the assessment of patients presenting with cognitive impairment. The National Institute for Health and Clinical Excellence (NICE) guidelines (2006: p. 10) recommend:

'Structural imaging should be used in the assessment of people with suspected dementia to exclude other cerebral pathologies and to help establish the subtype diagnosis. Magnetic resonance imaging (MRI) is the preferred modality to assist with early diagnosis and detect subcortical vascular changes, although computed tomography (CT) scanning could be used.'
However, NICE provides no clear guidance as to when neuroimaging is indicated, which means that there is wide variation in clinical practice in different areas of the country. The NICE guidelines recommend imaging in cases of earlyonset memory impairment, in patients with a short symptom onset and in those with neurological symptoms. This is to identify potentially reversible causes such as normal pressure hydrocephalus, subdural haemorrhage and brain tumour.

Table 1 outlines the basic details of CT, MRI, single-photon emission computed tomography (SPECT) and positron emission tomography (PET) in terms of indications, benefits, risks, adverse effects, costs and tolerability in the elderly.

There has been a greater clinical emphasis on functional neuroimaging, such as technetium99m hexamethyl propylene amine oxime (Tc-99m HMPAO) SPECT and DaTSCANs. Single-photon emission computed tomography uses radio-labelled tracers such as Tc-99m HMPAO to measure cerebral perfusion to show areas where blood flow is reduced. This procedure takes about $30 \mathrm{~min}$ to perform and can help in the differential diagnosis of dementia. DaTSCANs use a tracer that binds to the presynaptic dopamine transporter and is useful in the investigation of dementia with Lewy bodies and Parkinson's disease, in which there is decreased uptake in the transporters of the tracer ${ }^{123}$ I ioflupane.

\section{Alzheimer's disease}

In clinical diagnosis of Alzheimer's disease, NICE recommends that clinicians use the National Institute of Neurological and Communicative Disorders and Stroke and the Alzheimer's Disease and Related Disorders Association (NINCDSADRDA) diagnostic criteria (McKhann 1984) (Box 1). These criteria have a sensitivity of 0.93 (i.e. will correctly detect patients who have Alzheimer's disease), however, they only have a specificity of 0.23 , as patients with other forms of dementia often fulfil the criteria (Varma 1999).
Victoria Sullivan is an ST5 in forensic psychiatry in the North Western Deanery. Biswadeep Majumdar is a consultant in old age psychiatry in Mersey Care NHS Trust. Anna Richman is a consultant in old age psychiatry in Mersey Care NHS Trust. Sobhan Vinjamuri is a consultant in nuclear medicine at Royal Liverpool University Hospital.

Correspondence Dr Victoria Sullivan, Guild Lodge, Guild Park, Whittingham Lane, Goosnargh, Preston PR3 2JH, UK. Email: victoria. sullivan@doctors.org.uk 


\begin{tabular}{|c|c|c|c|c|}
\hline & CT & MRI & SPECT & PET \\
\hline Scan details & $\begin{array}{l}\text { X-rays producing cross-sectional } \\
\text { images }\end{array}$ & $\begin{array}{l}\text { Magnetic field alters the axes } \\
\text { of spinning protons, which is } \\
\text { detected by scanner }\end{array}$ & $\begin{array}{l}\text { Uses a gamma camera to detect } \\
\text { gamma-emitting radioisotopes } \\
\text { Can involve a CT scan at same } \\
\text { time }\end{array}$ & $\begin{array}{l}\text { Uses a gamma camera to detect } \\
\text { a positron-emitting radioisotope } \\
\text { CT scan can be performed during } \\
\text { same session }\end{array}$ \\
\hline Benefits & $\begin{array}{l}\text { Quick, painless, quiet, readily } \\
\text { available, well-established }\end{array}$ & $\begin{array}{l}\text { Non-invasive, painless, no } \\
\text { exposure to ionising radiation, } \\
\text { post-scan modification of images }\end{array}$ & $\begin{array}{l}\text { Allows assessment of blood flow } \\
\text { to brain } \\
\text { Relatively quick (about } 15 \mathrm{~min} \text { ) }\end{array}$ & $\begin{array}{l}\text { Allows assessment of biological } \\
\text { activity such as glucose activity } \\
\text { Non-invasive }\end{array}$ \\
\hline Cost & Least expensive & Slightly more expensive than CT & $\begin{array}{l}\text { Relatively inexpensive compared } \\
\text { with PET }\end{array}$ & $\begin{array}{l}\text { Most expensive, particularly if CT } \\
\text { used in combination }\end{array}$ \\
\hline Invasiveness & $\begin{array}{l}\text { Standard CT non-invasive } \\
\text { Contrast media can cause allergic } \\
\text { reactions }\end{array}$ & $\begin{array}{l}\text { Standard MRI non-invasive } \\
\text { Contrast media can cause allergic } \\
\text { reactions }\end{array}$ & $\begin{array}{l}\text { Intravenous injection, with rare } \\
\text { possibility of allergy }\end{array}$ & $\begin{array}{l}\text { Potential allergies to } \\
\text { radioisotopes (these are short- } \\
\text { lived isotopes) }\end{array}$ \\
\hline Risks & $\begin{array}{l}\text { Radiation exposure } \\
\text { Radiologist experience in } \\
\text { interpretation }\end{array}$ & $\begin{array}{l}\text { Contraindicated with some metal } \\
\text { implants }\end{array}$ & $\begin{array}{l}\text { Radiation exposure } \\
\text { Allergic reaction to radioisotope }\end{array}$ & $\begin{array}{l}\text { Exposure to ionising radiation - } \\
\text { substantial if concomitant CT }\end{array}$ \\
\hline $\begin{array}{l}\text { Tolerability in } \\
\text { elderly patients }\end{array}$ & Generally well tolerated & $\begin{array}{l}\text { Can be less well tolerated, as } \\
\text { longer to perform, claustrophobic } \\
\text { and noisy }\end{array}$ & $\begin{array}{l}\text { Can be less well tolerated } \\
\text { because of the invasiveness and } \\
\text { claustrophobia }\end{array}$ & Generally well tolerated \\
\hline
\end{tabular}

CT, computed tomography; MRI, magnetic resonance imaging; PET, positron emission tomography; SPECT, single-photon emission computed tomography.

\section{Computed tomography}

Computed tomography is a well-established, widely available, quick and relatively inexpensive tool. However, it is associated with radiation exposure, which can limit the use of serial scanning. It is most commonly used to exclude potentially reversible causes of cognitive impairment, but it can have some diagnostic value in Alzheimer's disease. Normal ageing (without cognitive impairment) is

BOX 1 NINCDS-ADRDA diagnostic criteria for probable Alzheimer's disease

For a diagnosis of probable Alzheimer's disease: $A$ plus one or more of supportive features $B, C, D$ or $E$

\section{Core diagnostic criteria}

A. Presence of an early and significant episodic memory impairment that includes the following features:

1 gradual and progressive change in memory function reported by patients or informants over a period of more than 6 months

2 objective evidence of significantly impaired episodic memory on testing: recall deficit that does not improve significantly or does not normalise with cues or recognition testing

3 the episodic memory impairment can be isolated or associated with other cognitive changes at the onset of Alzheimer's disease or as Alzheimer's disease advances. associated with general brain atrophy. Therefore, neuroimaging studies of brain volume show very little discrimination between normal ageing, cognitive impairment and dementia (Petrella 2003). However, there are several studies that show atrophy of specific brain regions on CT and MRI: notably, the hippocampus and entorhinal cortices in the medial temporal lobes are associated with cognitive impairment in Alzheimer's disease (O'Brien 2000). The sensitivity and specificity of using CT alone in detecting medial temporal lobe atrophy is $94 \%$ and 93\% respectively (O’Brien 2007).

\section{Magnetic resonance imaging}

Magnetic resonance imaging is becoming more widely used as a diagnostic tool in the investigation of dementia. Studies have shown that patients with Alzheimer's disease have a greater reduction in the volume of entorhinal cortices (37\%) and hippocampus (27\%) compared with normal elderly adults and patients with mild cognitive impairment (combined data: entorhinal cortices $11 \%$, hippocampus $13 \%$ ), and that these findings correlate well with neuropsychological testing (Davatzikos 2010). Although these changes are seen in up to two-thirds of cases of Alzheimer's disease, they are often absent on CT scans of patients with clinical diagnoses of the disease and are seen in about $5-10 \%$ of normal elderly individuals (O'Brien 2007). In patients over 80 years old, these changes are much less specific, and it may be more difficult to differentiate between normal ageing and Alzheimer's disease (O'Brien 2007). Therefore structural imaging (CT and MRI) is not routinely recommended in clinical diagnostic guidelines. 
Serial MRI has been used to assess the progression of changes in MRI findings; however, this has tended to be in clinical trial settings, with very little diagnostic applicability. In Alzheimer's disease, brain atrophy progresses at a rate of about $2 \%$ per year, in contrast to $0.1-0.5 \%$ per year in controls who are cognitively intact (O'Brien 2007), but it remains to be seen whether this could be used diagnostically. Some studies suggest that serial MRI, in combination with other markers such as cerebral spinal fluid biomarkers and pattern classification, may be useful in differentiating patients with mild cognitive impairment from patients with Alzheimer's disease by assessing the rate of neurodegeneration (Davatzikos 2011).

\section{Functional neuroimaging}

Functional neuroimaging - SPECT, PET, proton magnetic resonance spectroscopy (1H-MRS) and magnetic resonance volumetry (MRV) - can be as effective as post-mortem pathological examination in differentiating elderly patients with normal cognition from patients with Alzheimer's disease (Kantarci 2003).

\section{Single-photon emission computed tomography}

Single-photon emission computed tomography findings in Alzheimer's disease centre on the detection of hypoperfusion of the temporal and parietal brain regions (Kantarci 2003) (Fig. 1). Jagust et al (2001) compared clinical diagnosis, SPECT findings and post-mortem examination and found that SPECT increased the diagnostic certainty in patients who were clinically diagnosed with Alzheimer's disease from 84 to $92 \%$. This study found that a negative SPECT in these patients reduced the diagnostic certainty to $70 \%$.

\section{Positron emission tomography}

Positron emission tomography imaging uses Pittsburgh Compound-B (PiB) to detect amyloid deposition in Alzheimer's disease, which can be seen early in the disease process. As the disease progresses, amyloid deposits are less of a burden than neurodegeneration (Quigley 2010), so PET imaging may be more useful in detecting cases of Alzheimer's disease in the early stages. Pittsburgh Compound-B PET imaging is sensitive in detecting deposition of amyloid in Alzheimer's disease, but it is less specific, as $10-30 \%$ of cognitively intact elderly people have PiB-PET-detected amyloid deposition (Quigley 2010).

${ }^{(18)}$ F-fluorodeoxyglucose $\left({ }^{(18)}\right.$ FDG) PET detects glucose metabolism, and studies suggest that bilateral hypometabolism of the corpus callosum, temporoparietal and frontal association areas may

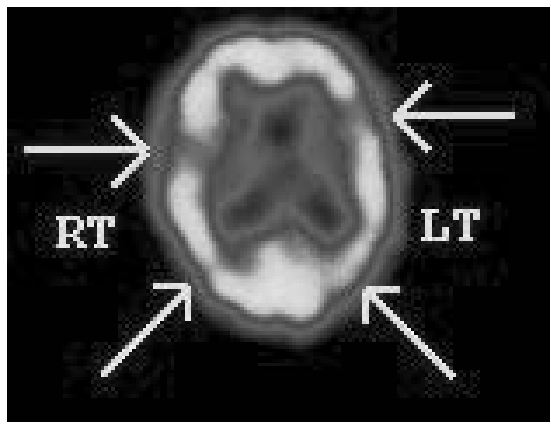

Tc-99m HMPAO single-photon emission computed tomography findings in a patient with clinical dementia. The scan shows reduced perfusion in the left frontotemporal, right temporal, left parietal and right parietal lobes. These findings suggest Alzheimer's disease.

be associated with Alzheimer's disease (Herholz 2007). Investigations using FDG PET can detect hypometabolism up to 1 year before subjective complaints of memory impairment in patients who later develop Alzheimer's disease (Herholz 2007).

\section{Future imaging techniques}

Future imaging techniques may centre on the use of more detailed MRI such as functional MRI (fMRI), spin-labelled MRI, magnetic resonance microscopy and magnetisation transfer MRI. Functional MRI measures cerebral blood flow in relation to functional activity in the brain and early studies show that inferior prefrontal and left temporal activation is associated with better scoring on cognitive testing (O’Brien 2007). However, fMRI is still in the early stages of trial for this use and is not a routine investigation. Advances in radioligands and tracers used in SPECT and PET scanning, such as monoclonal antibodies to beta-amyloid and flumazenil, may allow for further development in diagnostic validity.

\section{Vascular dementia}

To some extent vascular dementia may be preventable, as early detection of potentially modifiable vascular risk factors can allow for intervention to minimise subsequent cardiac and cerebrovascular disease. For the clinical diagnosis of vascular dementia, NICE (2006) recommends the National Institute of Neurological Disorders and Stroke and Association Internationale pour le Recherché et l'Enseignement en Neurosciences (NINDSAIREN) criteria (Box 2), which are widely used across the globe (Roman 1993). However, although the criteria have a high specificity at 0.80 , they have a relatively low sensitivity at 0.58 in diagnosing vascular dementia (Gold 1997). Therefore, the absence of these vascular lesions on CT or MRI excludes the diagnosis. 
BOX 2 Summary of the NINDS-AIREN criteria for the diagnosis of vascular dementia

\begin{tabular}{|c|c|}
\hline $\begin{array}{l}\text { - Dementia: } \\
\text { Memory impairment }\end{array}$ & $\begin{array}{l}3 \text { months of a stroke or stepwise } \\
\text { deterioration of cognitive deficits }\end{array}$ \\
\hline Deficits in two other cognitive domains & - Clinical features consistent with vascular \\
\hline $\begin{array}{l}\text { - Neurological signs of cerebrovascular } \\
\text { disease }^{\text {a }} \text { on neuroimaging: }\end{array}$ & $\begin{array}{l}\text { dementia: } \\
\text { Early gait disturbance }\end{array}$ \\
\hline Multiple large vessel infarcts/single & Frequent and unprovoked falls \\
\hline strategically placed infarct & Urinary symptoms not explained by \\
\hline Multiple basal ganglia and white matter & urological disease \\
\hline lacunes & Pseudobulbar palsy \\
\hline $\begin{array}{l}\text { Bilateral thalamic lesions or extensive } \\
\text { periventricular white matter lesions }\end{array}$ & Personality change, apathy and abulia \\
\hline $\begin{array}{l}\text { periventricular white matter lesions } \\
\text { - A relationship between the first two } \\
\text { disorders: onset of dementia within }\end{array}$ & $\begin{array}{l}\text { a. The NINDS-AIREN criteria propose that up } \\
\text { to } 25 \% \text { of white matter needs to be affected. } \\
\text { (After Roman 1993) }\end{array}$ \\
\hline
\end{tabular}

Importantly, O’Brien (2007) demonstrated that it was not possible to differentiate those with and without dementia using the diagnostic criteria and imaging results alone in older patients who had had a stroke. Therefore structural neuroimaging can only be used to support a clinical diagnosis.

\section{Periventricular white matter lesions}

Periventricular white matter lesions are an important component of the diagnostic criteria for vascular dementia (Box 2). However, these lesions are present in other forms of dementia, depression and even in normal ageing. It is felt that bilateral or left-sided white matter lesions may be important predictors of dementia and cognitive impairment (O’Brien 2000). Structural neuroimaging (CT/MRI) provides information about the volume, location and severity of vascular lesions and is an important tool for excluding other causes of cognitive impairment. Magnetic resonance imaging is more sensitive than CT in

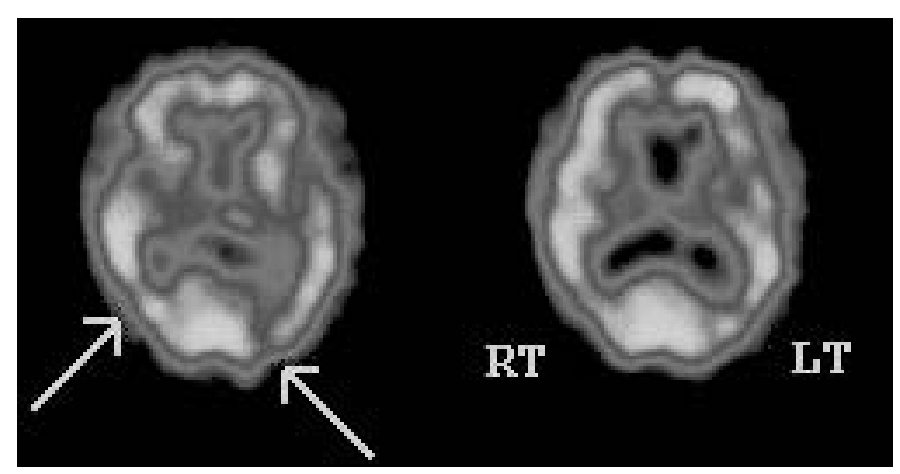

Tc-99m HMPAO single-photon emission computed tomography findings in a patient with cognitive impairment. The scan shows patchy perfusion defects in temporal and parietal lobes bilaterally, with reduction in perfusion to the occipital lobes (right > left). These findings are in keeping with vascular dementia. detecting ischaemic lesions (O’Brien 2007). It has been suggested that the presence of periventricular white matter changes can help differentiate vascular dementia from Alzheimer's disease, as both conditions are associated with cortical atrophy and ventricular enlargement on imaging (O’Brien 2000).

\section{Functional neuroimaging}

Technetium-99m HMPAO SPECT findings in vascular dementia reveal considerably reduced cerebral blood flow to certain brain areas compared with Alzheimer's dementia; a typical SPECT scan is shown in Fig. 2. These brain regions include frontal lobes and the basal ganglia. Other studies have demonstrated reduced cerebral blood flow in bilateral thalami, anterior cingulate gyri, superior temporal gyri, caudate and left parahippocampal gyrus in patients with vascular dementia compared with controls (Shim 2006). However, SPECT is not routinely recommended in the investigation of dementia owing to a lower diagnostic accuracy than that of clinical guidelines; sensitivity is reported to be as low as $43 \%$ (Shim 2006).

Positron emission tomography imaging in vascular dementia shows hypometabolism of cortical and subcortical brain areas. One PET study of patients with Alzheimer's disease and vascular dementia found deficits in metabolism in thalamus, caudate and frontal lobe which were strongly associated with vascular dementia (Pascual 2010).

\section{Dementia with Lewy bodies}

Dementia with Lewy bodies shares clinical symptoms with Alzheimer's and Parkinson's diseases (Box 3). It can be distinguished from Parkinson's disease in that the cognitive symptoms and Parkinsonian symptoms are relative and appear within 1 year of each other. Parkinson's disease can be diagnosed if cognitive symptoms appear more than 1 year after the onset of motor symptoms. Dementia with Lewy bodies has pathological changes which can separate it from Alzheimer's disease: the presence of Lewy bodies (which are neuronal inclusion bodies composed of alpha-synuclein and ubiquitin protein) and Lewy neurites. The International Consensus Criteria for the clinical diagnosis of probable dementia with Lewy bodies are shown in Box 4 (McKeith 1996); the criteria have low sensitivity and high specificity (Watson 2009).

\section{Computed tomography}

Computed tomography has been less useful in differentiating Alzheimer's disease from dementia 
with Lewy bodies. Watson and colleagues (2009) completed a review of MRI studies of patients who had dementia with Lewy bodies. They found the disease to be associated with less atrophy of the medial temporal lobes and principally subcortical atrophy; there was very little difference between patients with dementia with Lewy bodies and patients with Parkinson's disease dementia. This may allow a way of distinguishing dementia with Lewy bodies from Alzheimer's disease, where medial temporal lobe atrophy is predominant.

\section{Functional neuroimaging}

Single-photon emission computed tomography has been considered to be the gold standard scan in the diagnosis of dementia with Lewy bodies, showing reduced dopamine transporter activity in the basal ganglia. ${ }^{[123]}$ I-FP-CIT SPECT (DaTSCAN; Fig. 3) uses a ligand that binds to the dopamine transporter molecule; this can highlight areas of nigrostriatal degeneration that lead to dementia with Lewy bodies. Studies assessing this show specificity of up to $90 \%$ and sensitivity of $75 \%$ (Walker 2009), suggesting that DaTSCAN is more accurate than clinical diagnosis alone.

Positron emission tomography reveals hypometabolism in the occipital cortices (Walker 2009). Pittsburgh Compound-B PET shows amyloid deposition in dementia with Lewy bodies similar to that in Alzheimer's disease, but deposition is less in Parkinson's disease dementia than in dementia with Lewy bodies. This suggests that, although amyloid deposition is part of the pathology of the disease, it may have little effect on the clinical manifestation (Brooks 2009).

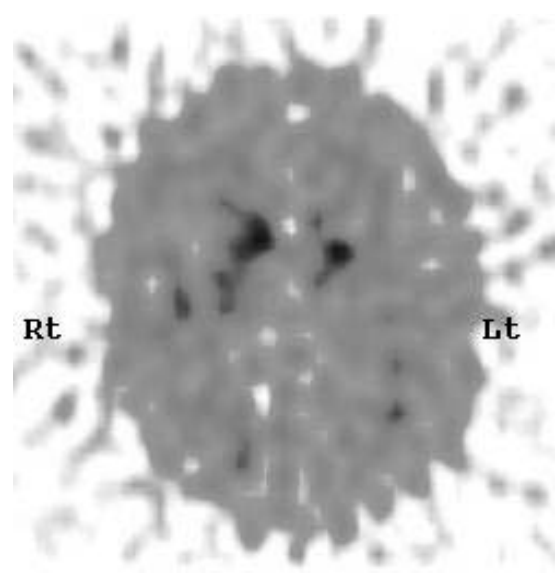

DaTSCAN for a patient with suspected dementia with Lewy bodies. The scan shows reduced tracer uptake in both putamina, with relatively normal uptake in the heads of caudate nuclei bilaterally. These findings are in keeping with dementia with Lewy bodies.

\section{BOX 3 Case vignette 1}

Mrs $\mathrm{Y}$, aged 78, was referred to a memory clinic with a 7-month history of deteriorating memory. She lived alone and her family had become concerned as she appeared less able to care for herself. She was more forgetful: misplacing things, forgetting appointments and meals. She had started to leave sweets around the house, and when asked about this she said that children often visited her during the day. She also spoke about seeing children playing in her garden. On further questioning, it emerged that Mrs $\mathrm{Y}$ had no grandchildren and her family thought it unlikely that any children visited her. Her confusion appeared to fluctuate, and some days it was better than others. Her sleep pattern had become quite disturbed and she frequently complained of nightmares. The family were concerned that she was having falls and appeared to be slow in her movements, having been a very sprightly woman before. There was no family history of dementia and no medical history.

Physical examination revealed bilateral cogwheel rigidity and bradykinesia. Her gait was unsteady. Mini-Mental State Examination (Folstein 1975) was 21/30 (losing 6 points on orientation and 3 points on recall). Addenbrooke's Cognitive Examination (Mathuranath 2000) score was $69 / 100$ (she lost 6 points on orientation and concentration, 15 points on memory, 5 points on language and 5 points on visuospatial skills). She was referred for a DaTSCAN, which revealed low dopamine transporter uptake in the brain's basal ganglia.

Comparisons of ${ }^{[123]}$ I-FP-CIT SPECT with ${ }^{(18)}$ F-FDG PET showed that the former had a greater diagnostic accuracy and effect size, but that both were useful in the assessment of suspected dementia with Lewy bodies (Lim 2009).

\section{Frontotemporal dementia}

The case vignette in Box 5 describes a typical presentation of the symptoms of frontotemporal dementia. The NICE guidance (2006) suggests using the Lund-Manchester criteria to diagnosis

BOX 4 International Consensus Criteria for the clinical diagnosis of probable and possible dementia with Lewy bodies

I The central feature required for a diagnosis of dementia with Lewy bodies is progressive cognitive decline of sufficient magnitude to interfere with normal social or occupational function. Prominent or persistent memory impairment may not necessarily occur in the early stages but is usually evident with progression. Deficits on tests of attention and of frontal-subcortical skills and visuospatial ability may be especially prominent.

II Two of the following core features are essential for a diagnosis of probable dementia with Lewy bodies, one is essential for possible dementia with Lewy bodies:

a fluctuating cognition, with pronounced variations in attention and alertness

b recurrent visual hallucinations, which are typically well formed and detailed c spontaneous motor features of Parkinsonism

III Features supportive of the diagnosis are:

- repeated falls

- syncope

- transient loss of consciousness

- neuroleptic sensitivity

- systematised delusions

- hallucinations in other modalities.

IV A diagnosis of dementia with Lewy bodies is less likely in the presence of:

- stroke disease, evident as focal neurological signs or on brain imaging

- evidence on physical examination and investigation of any physical illness, or other brain disorder, sufficient to account for the clinical picture.

(McKeith 1996, with permission) 
of this type of dementia. Box 6 shows these incorporated into the consensus diagnostic criteria for the disease (Neary 1998). Structural neuroimaging is generally unremarkable apart from showing cortical and subcortical atrophy in the frontal and temporal lobes, usually with enlarged ventricles (O'Brien 2007). Frontotemporal dementia can be differentiated from Alzheimer's disease by the pattern of atrophy that tends to spread from the anterior to posterior, whereas in Alzheimer's disease the atrophy is uniform (O'Brien 2007). In some cases, the atrophy may be localised to a specific lobe or lobar area. There are three subcategories of frontotemporal dementia: frontal variant frontotemporal dementia, semantic dementia and progressive non-fluent aphasia (Hodges 2001). In the last form, atrophy of the left temporal lobe is more pronounced than in the other subtypes (David 2009).

Functional neuroimaging has great diagnostic sensitivity and may prove beneficial even when the structural scan shows very little. Both SPECT and PET reveal hypometabolism and hypoperfusion of the frontal and temporal lobes. In contrast, in Alzheimer's disease there tends to be hypometabolism of the parietal lobes early on in dementia progression.

\section{Other dementias}

\section{Huntington's disease}

Huntington's disease is a hereditary condition associated with chorea, neuropsychiatric features and dementia of insidious onset. Computed tomography and MRI often show atrophy of the heads of the caudate nuclei. This is associated

\section{BOX 5 Case vignette 2}

$\mathrm{Mr}$ X, a 70-year-old retired accountant's clerk, was referred to the memory clinic with low mood and a 12-month history of a gradual decline in his poor short-term memory. He had been misplacing things and repeating his conversations. He had become more socially withdrawn over the preceding 6 months; his wife reported that he had lost interest in her and his friends and preferred to watch television all day. He had developed a strict routine of eating his meals at set times every day and insisting on the same food. If this routine was disrupted, he would become very irritable and would lose his temper. He had become coarser in his personality and was frequently rude to his guests. There was no family history of dementia. His medical history included hypertension and type 2 diabetes.

Physical examination was unremarkable and neurological examination was normal. MiniMental State Examination (Folstein 1975) score was 27/30 (losing 2 points on repetition and 1 on three-stage command). Addenbrookes Cognitive Examination (Mathuranath 2000) score was 74/100 (losing 5 points on memory, 10 points on verbal fluency, 8 on language and 3 on visuospatial skills). He was able to generate 7 animals in $1 \mathrm{~min}$ and 4 words beginning with ' $P$ '. There was some evidence of perseveration on examination and he performed poorly on proverb interpretation, cognitive estimates, Luria motor sequencing and Go-No-go testing. Magnetic resonance imaging revealed bilateral frontal lobe atrophy.
BOX 6 Consensus clinical diagnostic criteria for frontotemporal dementia

Core diagnostic features (all must be present)

- Insidious onset and gradual progression

- Early decline in social interpersonal conduct

- Early impairment in regulation of personal conduct

- Early emotional blunting

- Early loss of insight

Supportive diagnostic features

- Behavioural disorder

Decline in personal hygiene and personal grooming

Mental rigidity and inflexibility

Distractibility and impersistence

Hyperorality and dietary changes

Perseverative and stereotyped behaviour

Utilisation behaviour

- Speech and language: altered speech output (aspontaneity and economy of speech, press of speech), stereotypy of speech, echolalia, perseveration, mutism

- Physical signs: primitive reflexes, incontinence, akinesia, rigidity, tremor, low/labile blood pressure

- Investigations

Neuropsychology: impaired frontal lobe tests; no amnesia or perceptual deficits

ECG: normal on conventional ECG despite clinically evident dementia

Brain imaging: predominant frontal and/or anterior temporal abnormality

(Neary 1998, with permission)

with a loss of the normal convex bulging into the lateral walls of the frontal horns (David 2009). Caudate atrophy is highly specific to Huntington's disease. Magnetic resonance imaging studies have also shown subcortical atrophy and atrophy of the thalamus and medial temporal lobes, which appears to correlate with the degree of cognitive impairment (David 2009). Single-photon emission computed tomography and PET scans show decreased blood flow and glucose metabolism in the caudate nuclei (David 2009).

\section{Creutzfeldt-Jakob disease}

Creutzfeldt-Jakob disease (CJD) belongs to the group of transmissible spongiform encephalopathies, which also includes Gerstmann-SträusslerScheinker disease and fatal familial insomnia. These prion diseases are associated with relatively rapid onset of dementia together with other neuropsychiatric symptoms such as depression, anxiety and behavioural changes, cerebellar ataxia and myoclonus. Variant CJD is a distinct 
disease from sporadic CJD and is associated with characteristic signs on MRI, which have been incorporated into the World Health Organization's (2006) diagnostic criteria. Magnetic resonance imaging shows the distinctive pulvinar sign and hockey stick sign. The pulvinar sign is bilateral pulvinar hyperintensity, relative to the anterior putamen, on fluid attenuated inversion recovery (FLAIR) MRI (Collie 2003). The hockey stick sign is hyperintensity in the dorsomedial thalamic nuclei and periaqueductal grey matter (Collie 2003). Both SPECT and PET show regional brain hypometabolism and hypoperfusion, but these are fairly non-specific.

\section{Progressive supranuclear palsy}

Progressive supranuclear palsy is associated with frontotemporal dementia. Magnetic resonance image changes may include atrophy of the midbrain and brainstem, and third ventricle dilation, but these findings are not specific to progressive supranuclear palsy (Mishina 2004). Positron emission tomography studies in patients with frontotemporal dementia show a global hypometabolism, but this is more pronounced in the frontal lobes, caudate, putamen and thalamus (Mishina 2004).

\section{Mild cognitive impairment}

Patients with mild cognitive impairment are at risk of developing dementia. It has been considered to be a prodromal stage in the development of Alzheimer's disease: between 10 and $15 \%$ of patients with amnestic mild cognitive impairment with no vascular risk factors develop Alzheimer's disease each year (Ganguli 2004). Structural imaging of patients with clinical mild cognitive impairment has shown a reduction in the volumes of the entorhinal cortices and hippocampi, more than in the cognitively intact elderly, but less than that seen in Alzheimer's disease (O'Brien 2007). Serial MRI scanning of patients with mild cognitive impairment showed grey matter loss in the amygdala, entorhinal cortices and anterior hippocampus (O'Brien 2007). As cognition deteriorates and the clinical picture begins to resemble Alzheimer's disease, this grey matter loss extends to incorporate the entire hippocampi and temporal and parietal lobes (Whitwell 2007).

In studies of mild cognitive impairment, SPECT and PET reveal changes similar to those seen in Alzheimer's disease: hypoperfusion and hypometabolism of the temporal and parietal lobes (Whitwell 2007). Patients with mild cognitive impairment who subsequently developed Alzheimer's disease had more significant reductions in cerebral blood flow to the medial temporal lobes than those patients who did not develop the disease (Whitwell 2007).

Several studies have assessed the utility of neuroimaging in the prediction of conversion from mild cognitive impairment to Alzheimer's disease. These found that a smaller volume of the entorhinal cortices and hippocampi both independently predicted conversion to Alzheimer's disease (Devanand 2007). However, there was little additional benefit from this than for measuring cognitive variables and predicting based on age.

\section{Special groups}

\section{Intellectual disability}

The prevalence of dementia in people with intellectual disability is higher than in the general population. Down syndrome in particular is associated with Alzheimer's disease: about 54.5\% of 60- to 69-year-olds with the syndrome have a diagnosis of dementia (Stanton 2004). Intellectual disability not due to Down syndrome is also associated with a higher prevalence of dementia: about $13 \%$ of over-50-year-olds and $22 \%$ of over65-year-olds in this group have a form of dementia (Moss 1993). The diagnosis of dementia in people with intellectual disability can be more complex because of difficulties in detecting cognitive decline and because changes in behaviour are often attributed to the intellectual disability. Consequently, patients often present to dementia services late in the progression of the disease (Stanton 2004).

The use of neuroimaging in dementia in patients with intellectual disability has not been studied to a great extent. Most of the research has focused on Down syndrome and has involved small numbers of patients. Structural imaging (CT and MRI) has shown atrophy of the medial temporal lobes, in keeping with the changes expected in Alzheimer's disease (Stanton 2004). However, it must be borne in mind that people with Down syndrome and other forms of intellectual disability are likely to have abnormal scans anyway, and so scans may be misinterpreted (Strydom 2002). Consequently, neuroimaging may be used as a supportive tool in examining these patients for dementia, but diagnosis remains a task for experienced clinicians.

\section{Early-onset dementia}

A 2007 report for the Alzheimer's Society (King's College London 2007) revealed that early-onset dementia (diagnosed before the age of 65 ) accounted for $2.2 \%$ of cases of dementia in the country. Alzheimer's disease was the most common subtype of dementia in patients of any age. Late-onset dementia was slightly more 
common in women; among patients aged 50-65, early-onset dementia was more common in men, with frontotemporal dementia accounting for a substantial proportion of cases. In Black and minority ethnic groups, $6.1 \%$ cases of dementia were of early onset, compared with the national average of $2.2 \%$.

Research into neuroimaging findings in earlyonset dementia is sparse, and there are very few studies that make comparisons between earlyonset and late-onset findings. One study that compared MRI findings, in addition to neuropsychology battery results, in patients with earlyonset and late-onset Alzheimer's disease found that in early-onset disease cortical atrophy was more common in occipital and parietal lobes, whereas in late-onset disease hippocampal atrophy was more common (Frisoni 2007). Comparison of TC-99m HMPAO SPECT scans of patients with early- and late-onset Alzheimer's disease showed that earlyonset disease was associated with hypoperfusion predominantly in the posterior cortical association area, whereas late-onset disease was associated with medial temporal lobe hypoperfusion (Kemp 2003). Although the numbers of patients in both of these studies were small, there do appear to be differences in neuroimaging findings between early-onset and late-onset dementia cases, and further research may be beneficial.

\section{Depressive pseudodementia}

Depressive pseudodementia is a reversible cause of cognitive impairment caused by depression. As depression is frequently comorbid with dementia, it can be difficult to distinguish between pseudodementia and a primary diagnosis of dementia. Magnetic resonance imaging findings in patients with either depression or dementia both show hippocampal atrophy, but there is a good correlation between the length of history of cognitive impairment and extent of atrophy of the entorhinal cortices (O'Brien 2000). For patients with cognitive dysfunction of unclear aetiology, the degree of temporal lobe atrophy on MRI could allow differentiation between dementia and depression: the former would show a greater degree of atrophy (O’Brien 2000).

A small study that evaluated SPECT findings in four samples - individuals with Alzheimer's disease, those with depression without cognitive impairment, those with depressive pseudodementia and healthy controls - found that the depressive pseudodementia group showed hypoperfusion of the temporoparietal region, similar to the findings in the Alzheimer's disease group, but different from findings in the depression group (Cho 2002).

\section{Discussion}

The question as to whether to use neuroimaging in the investigation and diagnosis of dementia is complicated. Largely, the diagnosis of dementia is a clinical one and the diagnostic criteria for the various subcategories of dementia place neuroimaging in a supportive role (Roman 1993; Neary 1998; Varma 1999; Watson 2009).

\section{Alzheimer's disease}

In the UK, Alzheimer's disease is the most common form of dementia, accounting for up to $60 \%$ of cases (Blennow 2006). Therefore, the utility of imaging in these cases is paramount. For Alzheimer's disease, the findings in CT and MRI are often seen in other conditions and are not necessarily specific to Alzheimer's disease. The characteristic signs on CT and MRI in Alzheimer's disease are frequently absent in clinical practice and can be seen in the elderly people without cognitive impairment. Therefore, at present, we would not suggest routinely screening patients with a clinical diagnosis of Alzheimer's disease using CT and MRI. Although PET and SPECT in Alzheimer's disease can be more sensitive and specific, the additional costs, invasiveness, radiation exposure, potential for allergic reactions and relatively poor tolerability of some of these procedures in the elderly limit their usefulness. Some centres require a CT scan to be performed before a SPECT or PET in order to increase sensitivity and rule out other causes for memory loss such as tumour. We would recommend the use of SPECT and PET only if there is clear diagnostic uncertainty.

\section{Vascular dementia}

For vascular dementia there is a clearer position for structural imaging; the NINDS-AIREN diagnostic criteria (Roman 1993) include the presence of vascular insults, including infarcts, basal ganglia and white matter lacunes or extensive periventricular white matter lesions (up to $25 \%$ of the white matter should be affected). Absence of these features, according to these criteria, excludes the diagnosis. Clearly, to detect the presence or absence of these lesions, there needs to be some form of imaging performed. Computed tomography can effectively detect large vessel infarction or ischaemia, but MRI is more sensitive in detecting extensive white matter lesions. Diagnosis of vascular dementia is often made on clinical grounds, but where there is uncertainty, CT or MRI should be used according to local resources and patient tolerability and contraindications; SPECT and PET should be used only 
if the diagnosis is in doubt and CT or MRI has been inconclusive.

\section{Other types of dementia}

For less common types of dementia, imaging may be indicated. For dementia with Lewy bodies, CT and MRI are less useful in differentiating it from other subtypes of dementia, particularly from Alzheimer's disease. The gold standard form of imaging in these patients is a SPECT scan with a radioligand that binds to the dopamine transporter molecule (DaTSCAN) and demonstrates nigrostriatal degeneration. As SPECT has been shown to have greater diagnostic accuracy than PET in dementia with Lewy bodies, we would recommend SPECT as an adjunct to clinical diagnosis in patients suspected of having this type of dementia.

Functional neuroimaging also plays a more important role in the diagnosis of frontotemporal dementia. Computed tomography and MRI findings are difficult to distinguish from changes seen in Alzheimer's disease, whereas PET shows hypometabolism and hypoperfusion of the frontal and temporal lobes more specifically. The diagnosis is principally based on symptoms and signs, and imaging should be reserved for cases where the diagnosis is in doubt.

Huntington's disease and CJD both have characteristic imaging findings: caudate atrophy and pulvinar sign/hockey stick sign respectively. These findings are more likely to be present on MRI and in suspected cases imaging can be used to support the diagnosis, which is in the most part dependent on genetic testing for Huntington's disease and biopsy for CJD. Single-photon emission computed tomography and PET will add little to the investigation and diagnosis of these conditions.

\section{Recommendations}

Owing to wide variation in clinical practice across the UK, we contacted NICE to clarify the recommendations in the dementia guidelines (National Institute for Health and Clinical Excellence 2006) to determine whether all patients with suspected dementia should undergo neuroimaging. This was their response:

\begin{abstract}
'Scanning should be done when the diagnosis is unsure, or when the clinician needs to establish a subtype or where there is uncertainty about other cerebral pathology. If the clinician has patients in which the diagnosis on clinical grounds is absolutely obvious, a scan will not be necessary' (K. Jackson, 2010, personal communication).
\end{abstract}

The answer to the question 'To scan or not to scan?' in suspected dementia and mild cognitive impairment is that routine scanning is not indicated in all patients and healthcare professionals should use their clinical judgement. The diagnosis of dementia should be made by experienced specialist clinicians, based primarily on history and mental state examination. In keeping with NICE guidance and also in our opinion, the use of diagnostic criteria can help discriminate between the subtypes of dementia. Routine imaging such as CT and MRI should not be used. Imaging should be reserved for cases where there is uncertainty about the diagnosis. Usually, PET and SPECT are relatively less well tolerated than CT among elderly people, so they should be reserved for complex cases, patients with suspected dementia with Lewy bodies and in cases where further diagnostic clarity is required.

\section{References}

Blennow K, de Leon MJ, Zetterberg H (2006) Alzheimer's disease. Lancet 368: 387-403.

Brooks DJ (2009) Imaging amyloid in Parkinson's disease dementia and dementia with Lewy bodies with positron emission tomography. Movement Disorders 24 (suppl 2): S742-7.

Cho MJ, Lyoo IK, Lee DW, et al (2002) Brain single photon emission computed tomography findings in depressive pseudodementia patients. Journal of Affective Disorders 69: 159-66.

Collie DA, Summers DM, Sellar RJ, et al (2003) Diagnosing variant Creutzfeldt-Jakob disease with the pulvinar sign: MR imaging findings in 86 neuropathologically confirmed cases. American Journal of Neuroradiology 24: 1560-9.

Davatzikos C, Bhatt P, Shaw LM, et al (2011) Prediction of MCI to $\mathrm{AD}$ conversion, via MRI, CSF biomarkers and pattern classification. Neurobiology of Aging 32: 2322.e19-27.

David AS, Fleminger S, Kopelman MD, et al (2009) Alzheimer's disease and other dementias. In Lishman's Organic Psychiatry: A Textbook of Neuropsychiatry (4th edn): 543-615. Wiley-Blackwell.

Devanand DP, Pradhaban G, Liu X, et al (2007) Hippocampal and entorhinal atrophy in mild cognitive impairment: prediction of Alzheimer disease. Neurology 68: 828-36.

Folstein MF, Folstein SE, McHugh PR (1975) 'Mini-mental state'. A practical method for grading the cognitive state of patients for the clinician. Journal of Psychiatric Research 12: 189-98.

Frisoni GB, Pievani M, Testa C, et al (2007) The topography of grey matter involvement in early and late onset Alzheimer's disease. Brain 130 (pt 3): 720-30.

Ganguli M, Dodge HH, Shen C, et al (2004) Mild cognitive impairment, amnestic type: an epidemiologic study. Neurology 63: 115-21.

Gold G, Giannakopoulos P, Montes-Paixao JC, et al (1997) Sensitivity and specificity of newly proposed clinical criteria for possible vascular dementia. Neurology 49: 690-4.

Herholz K, Carter SF, Jones M (2007) Positron emission tomography imaging in dementia. British Journal of Radiology 80 (Spec No 2): S160-7.

Hodges JR (2001) Frontotemporal dementia (Pick's disease): clinical features and assessment. Neurology 56 (suppl 4): S6-S10.

Jagust W, Thisted R, Devous S, et al (2001) SPECT perfusion imaging in the diagnosis of Alzheimer's Disease. Neurology 56: 950-6.

Kantarci K, Jack Jr CR (2003) Neuroimaging in Alzheimer disease: an evidence-based review. Neuroimaging Clinics of North America 13: 197-209.

Kemp PM, Holmes C, Hoffman SMA, et al (2003) Alzheimer's disease: differences in technetium-99m HMPAO SPECT scan findings between early onset and late onset dementia. Journal of Neurology, Neurosurgery and Psychiatry 74: 715-9.
MCO answers

1 b $\quad 2$ a $\quad 3$ e $\quad 4 d \quad 5$ a 
King's College London, London School of Economics (2007) Dementia UK: The Full Report. Alzheimer's Society.

Lim SM, Katsifis A, Villemange A, et al (2009) The 18F-FDG PET Cingulate Island sign and comparison to [123]|-Beta-CIT SPECT for diagnosis of dementia with Lewy bodies. Journal of Nuclear Medicine 50: 1638-45.

Mathuranath PS, Nestor PJ, Berrios GE, et al (2000) A brief cognitive test battery to differentiate Alzheimer's disease and frontotemporal dementia. Neurology 55: 1613-20.

McKeith IG, Galasko D, Kosaka K, et al (1996) Consensus guidelines for the clinical and pathologic diagnosis of dementia with Lewy bodies (DLB): report of the Consortium on DLB international workshop. Neurology 47 : $1113-24$

McKhann G, Drachman D, Folstein M, et al (1984) Clinical diagnosis of Alzheimer's disease: report of the NINCDS-ADRDA Work Group under the auspices of Department of Health and Human Services Task Force on Alzheimer's Disease. Neurology 34: 939-44.

Mishina M, Ishii K, Mitani K, et al (2004) Midbrain hypometabolism as early diagnostic sign for progressive supranuclear palsy. Acta Neurologica Scandinavica 110: 128-35.

Moss S, Patel P (1993) The prevalence of mental illness in people with intellectual disability over 50 years of age, and the diagnostic importance of information from carers. Irish Journal of Psychological Medicine 14: $110-29$

National Institute for Health and Clinical Excellence (2006) Dementia: Supporting People with Dementia and their Carers in Health and Social Care (Clinical Guideline CG42). NICE.

Neary D, Snowden JS, Gustafson L, et al (1998) Frontotemporal lobar degeneration: a consensus on clinical diagnostic criteria. Neurology 51 : 1546-54.

O'Brien JT, Barber B (2000) Neuroimaging in dementia and depression. Advances in Psychiatric Treatment 6: 109-19.

O'Brien JT (2007) Role of imaging techniques in the diagnosis of dementia. British Journal of Radiology 80: S71-7.

Office for National Statistics (2012) Ageing in the UK Datasets - 2011 Pensions Act Update. ONS (http://www.ons.gov.uk/ons/publications/rereference-tables.html?edition=tcm\%3A77-248389).
Pascual B, Prieto E, Arbizu J, et al (2010) Brain glucose metabolism in vascular white matter disease with dementia: differentiation from Alzheimer disease. Stroke 41: 2889-93.

Petrella JR, Coleman RE, Doraiswamy PM (2003) Neuroimaging and early diagnosis of Alzheimer's disease: a look into the future. Radiology 226: 315-36.

Quigley H, Colloby S, O'Brien T (2010) PET imaging of brain amyloid in dementia: a review. International Journal of Geriatric Psychiatry 26 991-9.

Roman GC, Tatemichi TK, Erkinjuntti T, et al (1993) Vascular dementia: diagnostic criteria for research studies. Report of the NINDS-AIREN International Workshop. Neurology 43: 250-60.

Shim YS, Yang DW, Kim BS, et al (2006) Comparison of regional cerebral blood flow in two subsets of subcortical ischaemic vascular dementia: statistical parametric mapping analysis of SPECT. Journal of Neurological Sciences 250: 85-91.

Stanton LR, Coetzee RH (2004) Down's syndrome and dementia. Advances in Psychiatric Treatment 10: 50-8

Strydom A, Hassiotis A, Walker Z (2002) Clinical use of structural magnetic resonance imaging in the diagnosis of dementia in adults with Down's syndrome. Irish Journal of Psychological Medicine 19: 60-3.

Varma AR, Snowden JS, Lloyd JJ, et al (1999) Evaluation of the NINCDS-ADRDA criteria in the differentiation of Alzheimer's disease and frontotemporal dementia. Journal of Neurology, Neurosurgery and Psychiatry 66: 184-8.

Walker RWH, Walker Z (2009) Dopamine transporter single photon emission computerized tomography in the diagnosis of dementia with Lewy bodies. Movement Disorders 24 (suppl 2): S754-9.

Watson R, Blamire AM, O'Brien JT (2009) Magnetic resonance imaging in Lewy body dementias. Dementia and Geriatric Cognitive Disorders 28 : 493-506.

Whitwell JL, Przybelski SA, Weigand SD, et al (2007) 3D maps from multiple MRI illustrate changing atrophy patterns as subjects progress from mild cognitive impairment to Alzheimer's disease. Brain 130: 1777-86.

World Health Organization (2006) WHO Guidelines on Tissue Infectivity Distribution in Transmissible Spongiform Encephalitis (WL 300). WHO.

\section{MCOs}

Select the single best option for each question stem

\section{In Alzheimer's disease:}

a NICE recommends the NINDS-AIREN diagnostic criteria

b MRI shows atrophy of the entorhinal cortices and hippocampi

c CT and MRI changes are absent in up to $90 \%$ of cases

d PiB-PET is unable to detect amyloid deposition

e NICE recommends routine use of SPECT in investigating Alzheimer's disease.

2 In vascular dementia:

a NICE recommends the NINDS-AIREN diagnostic criteria

b periventricular grey matter lesions are essential for diagnosis

c PET shows hypermetabolism of cortical and subcortical brain areas

d MRI is less sensitive than CT in detecting ischaemia

e SPECT is recommended as a routine investigation
3 As regards dementia with Lewy bodies and frontotemporal dementia:

a dementia with Lewy bodies is diagnosed when Parkinsonism and dementia appear within 1 month of each other

b Lewy bodies are composed of ubiquitin and amyloid

c in dementia with Lewy bodies, SPECT shows hypermetabolism and hyperperfusion of the occipital lobes

d in frontotemporal dementia, atrophy spreads from posterior to anterior

e in frontotemporal dementia, SPECT and PET show hypometabolism and hypoperfusion of the frontal and temporal lobes.

\section{As regards other dementias:}

a CT and MRI in CJD show caudate atrophy

b the pulvinar sign is pulvinar hypointensity as seen on FLAIR MRI

c the hockey stick sign is specific to Huntington's disease

d progressive supranuclear palsy is associated with midbrain and brainstem atrophy e the hockey stick sign is hypointensity in the dorsomedial thalamic nuclei and periaqueductal grey matter.

5 Mild cognitive impairment:

a is considered to be a prodromal stage before the development of Alzheimer's disease

b is associated with normal volumes of the entorhinal cortices and hippocampi

c SPECT and PET show hypometabolism and hypoperfusion of the temporal and parieta lobes, but more than that seen in Alzheimer's disease

d patients who progress to Alzheimer's disease have less deficit of perfusion to the medial temporal lobes

e NICE recommends routine $\mathrm{CT}$ in the investigation of all patients with mild cognitive impairment. 